\title{
A percepção de mulheres diante da prevenção do câncer de colo de útero e a
}

\section{realização do exame Papanicolau}

The perception of women regarding the prevention of cervical cancer and the performance of the

\section{Pap smear}

La percepción de las mujeres sobre la prevención del cáncer de cuello uterino y la realización del

\section{Papanicolaou}

Recebido: 13/09/2021 | Revisado: 19/09/2021 | Aceito: 22/09/2021 | Publicado: 24/09/2021

João Felipe Tinto Silva

ORCID: https://orcid.org/0000-0003-3662-6673 Centro Universitário de Ciências e Tecnologia do Maranhão, Brasil

E-mail: felipetinto99@gmail.com

Maria Dhescyca Ingrid Silva Arruda

ORCID: https://orcid.org/0000-0002-9073-7844

Faculdade São Francisco da Paraiba, Brasil

E-mail: dhescycaingrid20@gmail.com

Iracema Silva da Costa

ORCID: https://orcid.org/0000-0003-3213-9325

Centro Universitário de Ciências e Tecnologia do Maranhão, Brasil

E-mail: iracemar589@gmail.com

Amanda Altino da Cruz

ORCID: https://orcid.org/0000-0002-1991-4940

Centro Universitário de Ciências e Tecnologia do Maranhão, Brasil

E-mail: amandacruz81126@gmail.com

Emanuel Osvaldo de Sousa

ORCID: https://orcid.org/0000-0003-2825-4275

Universidade Estadual do Piauí, Brasil

E-mail: emanfisio@hotmail.com

Diego Bruno Brito Cerqueira

ORCID: https://orcid.org/0000-0002-8083-1634 Faculdade Integral Diferencial, Brasil

E-mail: diegocerqueirafisioterapeuta@hotmail.com

Layanne Cavalcante de Moura

ORCID: https://orcid.org/0000-0003-2781-1076

Faculdade Integral Diferencial, Brasil

E-mail: layannecavalcante@ hotmail.com

Lucyanna Cavalcante de Moura

ORCID: https://orcid.org/0000-0002-7163-0339

Universidade Estadual do Maranhão, Brasil

E-mail: lucyanna2@hotmail.com

Victória Maria Pontes Martins

ORCID: https://orcid.org/0000-0002-8281-0132

Centro Universitário INTA, Brasil

E-mail: victoriapontes2014@ hotmail.com

Lucília da Costa Silva

ORCID: https://orcid.org/0000-0001-9386-5684

Centro Universitário Santo Agostinho, Brasil

E-mail: luciliafisio@outlook.com

Robson Feliciano da Silva

ORCID: https://orcid.org/0000-0003-4387-2469

Centro Universitário FACOL, Brasil

E-mail: robsonf.silva@unifacol.edu.br

Laís Goldner Barcellos

ORCID: https://orcid.org/0000-0002-0236-257X Faculdade Brasileira-MULTIVIX, Brasil

E-mail: laisgoldnerb@gmail.com

Lorena Esmeralda Nascimento Celeste

ORCID: https://orcid.org/0000-0001-8217-620X

Universidade Salvador, Brasil

E-mail: lory.celeste@yahoo.com.br 
Letícia Freitas de Castro Silva ORCID: https://orcid.org/0000-0001-5982-4096 Centro Universitário de Belo Horizonte, Brasi E-mail: leticiafreitascsilva@gmail.com

Elisa Pinheiro Weber

ORCID: https://orcid.org/0000-0002-1881-4673 Centro Universitário de Belo Horizonte, Brasil E-mail: elisapweber@gmail.com

Lana Cristina Pedrosa Ferreira ORCID: https://orcid.org/0000-0002-2226-5873 Centro Universitário de Belo Horizonte, Brasil E-mail: lanafferreira@gmail.com

Ana Paula Falcão de Morais ORCID: https://orcid.org/0000-0002-4840-6849 Centro Universitário de Belo Horizonte, Brasil

E-mail: anapaulafalcaomorais@hotmail.com

Mariana Ferreira Coelho

ORCID: https://orcid.org/0000-0003-4327-6536

Centro Universitário de Belo Horizonte, Brasil

E-mail: marianafc.2000@ hotmail.com

Fernanda Vieceli de Melo

ORCID: https://orcid.org/0000-0003-4280-9919 Centro Universitário de Belo Horizonte, Brasil

E-mail: fernandaviecelidemelo@gmail.com

Maiara Rodrigues Maia

ORCID: https://orcid.org/0000-0002-6719-2911

Hospital Sírio Libanês, Brasi

E-mail: maiara.enfermagem@hotmail.com

Samuel Carlos Tomaz

ORCID: https://orcid.org/0000-0003-3885-8857 Universidade Regional do Cariri, Brasil E-mail: samueltomaz47@yahoo.com

Marks Passos Santos

ORCID: https://orcid.org/0000-0003-1180-404X Faculdade Ages de Medicina, Brasil

E-mail: enfer.marks@hotmail.com

Bárbara Bispo de Santana

ORCID: https://orcid.org/0000-0002-7017-2401

Faculdade Ages de Senhor do Bonfim, Brasil

E-mail: prof.enf.barbarabispo@gmail.com

Geísa de Morais Santana

ORCID: https://orcid.org/0000-0001-8008-888X Universidade Estadual do Piauí, Brasil

E-mail: geisasantana97@gmail.com

Antônio Lucas Farias da Silva

ORCID: https://orcid.org/0000-0001-8010-1714 Centro Universitário UniFacid, Brasil E-mail: lucas1992farias@gmail.com

Vitória Gabriele Barros de Araújo

ORCID: https://orcid.org/0000-0003-2232-405X Universidade Estadual do Piauí, Brasil E-mail: gabriiellearaujo@ hotmail.com

Ellen Larissa Santos da Rocha Maciel

ORCID: https://orcid.org/0000-0001-5738-7822

Universidade Federal do Maranhão, Brasil E-mail: ellenlarissamaciel@gmail.com

Saulo Leite de Paula

ORCID: https://orcid.org/0000-0002-9511-6115 Universidade Federal do Ceará, Brasil E-mail: saulolp@yahoo.com.br

Ramon Lima Silva

ORCID: https://orcid.org/0000-0002-2454-7276 Faculdade Estácio de Teresina, Brasil E-mail: ramonaespi@ hotmail.com

\section{Resumo}

O presente estudo teve como objetivo descrever a percepção de mulheres acerca da prevenção do câncer de colo do útero e a realização do exame Papanicolau. Trata-se de uma revisão integrativa da literatura, onde o levantamento do estudo foi realizado entre julho e agosto de 2021, através das bases de dados MEDLINE, LILACS e BDENF. Utilizaram-se os Descritores em Ciências da Saúde (DeCS): Neoplasias do Colo do Útero, Teste de Papanicolaou e 
Saúde da Mulher. Após os critérios de inclusão e exclusão e análise dos estudos, 16 estudos foram selecionados para compor o presente estudo. O estudo mostra que as mulheres atribuem o exame de Papanicolau como fundamental para a prevenção e detecção precoce para doenças, no entanto, apresentam conhecimento insuficiente sobre o exame Papanicolau, fato este que está relacionado ao baixo nível de escolaridade e a baixa renda, e que as mesmas apresentam alguns sentimentos negativos como: dor, desconforto, constrangimento durante o exame, medo do resultado, ansiedade, nervosismo, vergonha, frustração e desconforto em realiza-lo. é indispensável as orientações pelos profissionais da saúde sobre o exame Papanicolau às mulheres que buscam os serviços de atenção básica. Devendo estes, realizar ações de educação em saúde de forma continuada, enfatizando a importância da realização periódica do exame Papanicolau pelas mulheres, proporcionando uma maior adesão ao exame, além de possibilitar uma assistência humanizada, contínua e de qualidade.

Palavras-chave: Neoplasias do colo do útero; Teste de Papanicolaou; Saúde da mulher.

\begin{abstract}
This study aimed to describe the perception of women about the prevention of cervical cancer and the performance of the Pap smear. This is an integrative literature review, where the survey of the study was carried out between July and August 2021, through the MEDLINE, LILACS and BDENF databases. The Health Sciences Descriptors (DeCS) were used: Cervical Neoplasms, Pap smear and Women's Health. After the inclusion and exclusion criteria and analysis of the studies, 16 studies were selected to compose the present study. The study shows that women attribute the Pap smear as fundamental for the prevention and early detection of diseases, however, they have insufficient knowledge about the Pap smear, a fact that is related to low education and low income, and that they have some negative feelings such as: pain, discomfort, embarrassment during the exam, fear of the result, anxiety, nervousness, shame, frustration and discomfort in taking the exam. The guidance of health professionals about the Pap smear for women who seek primary care services is essential. They should carry out health education actions on a continuous basis, emphasizing the importance of taking periodic Pap smears by women, providing greater adherence to the test, in addition to enabling humanized, continuous and quality care.
\end{abstract}

Keywords: Cervical neoplasms; Pap smear test; Women's health.

\title{
Resumen
}

Este estudio tuvo como objetivo describir la percepción de las mujeres sobre la prevención del cáncer de cuello uterino y la prueba de Papanicolaou. Se trata de una revisión integradora de la literatura, cuyo relevamiento del estudio se realizó entre julio y agosto de 2021, a través de las bases de datos MEDLINE, LILACS y BDENF. Se utilizaron los Descriptores de Ciencias de la Salud (DeCS): Neoplasias Cervicales, Papanicolaou y Salud de la Mujer. Después de los criterios de inclusión y exclusión y el análisis de los estudios, se seleccionaron 16 estudios para componer el presente estudio. El estudio muestra que las mujeres atribuyen el Papanicolaou como fundamental para la prevención y detección precoz de enfermedades, sin embargo, tienen un conocimiento insuficiente sobre el Papanicolaou, hecho que se relaciona con el bajo nivel de educación y bajos ingresos, y que tienen algunos aspectos negativos sentimientos como: dolor, malestar, vergüenza durante el examen, miedo al resultado, ansiedad, nerviosismo, vergüenza, frustración e incomodidad durante el examen. La orientación de los profesionales de la salud sobre las pruebas de Papanicolaou para las mujeres que buscan servicios de atención primaria es esencial. Deben realizar acciones de educación en salud de manera continua enfatizando la importancia del Papanicolaou periódica por parte de las mujeres, brindando mayor adherencia a la prueba, además de posibilitar una atención humanizada, continua y de calidad.

Palabras clave: Neoplasias cervicales; Prueba de Papanicolaou; La salud de la mujer.

\section{Introdução}

O Instituto Nacional de Controle do Câncer (INCA) estimou no ano de 2020, 16.590 casos de Câncer de Colo do Útero (CCU) no Brasil, sendo recomendado o rastreamento por meio de exame citológico com técnica de Papanicolau que é indicada para todas as mulheres sexualmente ativas, independentemente da idade. É considerado o terceiro câncer mais frequentes em mulheres no país e mesmo com o aumento das ações preventivas, controle populacional e desenvolvimento do tratamento, a taxa de mortalidade ainda não sofreu significativas reduções (Inca, 2020; Colares et al., 2020).

O exame citopatológico é oferecido pelo Sistema Único de Saúde (SUS), como parte da Atenção Primária à Saúde (APS) e das políticas de saúde da mulher, para realizar o rastreamento, o diagnóstico e o tratamento do CCU. Nas Unidades Básicas de Saúde (UBS) os enfermeiros identificam as mulheres elegíveis para a realização do exame, por meio de protocolos de priorização de usuárias, e buscam aquelas que não comparecem, oferecendo suporte e informação. Isso tem feito com que o 
número de exames tenha aumentado em pelo menos cinco pontos percentuais. O nível dos serviços oferecidos pelas UBS é fundamental para a adesão à realização do exame, principalmente em colaboração com a Estratégia Saúde da Família, cujos profissionais estão mais próximos das mulheres (Santos, Silveira \& Rezende, 2019).

O procedimento do exame de prevenção contra o CCU, ou também conhecido como Papanicolau, é realizado com o esfregaço das células proveniente da ectocérvice e da endocérvice, que são retirados por raspagem do colo do útero. É considerado um procedimento prático e de baixo custo para o rastreamento do câncer de colo de útero. Sendo este um problema de saúde pública, devido à alta ocorrência, ocupar o terceiro lugar entre os tipos de câncer mais frequente entre as mulheres, ressalvando o câncer de pele melanoma, ficando atrás do câncer de mama e do colorretal (Gurgel et al., 2019).

A literatura aponta que os principais fatores de riscos que podem abranger o desenvolvimento deste câncer são o início precoce da prática sexual, múltiplos parceiros, a classe social socioeconômica baixa, multiparidade, o uso de contraceptivos orais, tabagismo, dentre os quais o vírus do papiloma vírus (HPV) é o principal fator de risco na transmissão dessa doença (Souza \& Costa, 2015).

O CCU se caracteriza através da replicação do epitélio que reveste o útero comprometendo os tecidos subjacentes, podendo ou não atingir órgãos e estruturas na região. Existem dois tipos de carcinomas invasores que variam de acordo com a origem do epitélio comprometido, nesta classe, temos: o carcinoma epidermoide, ocorre com mais incidência e atinge o epitélio escamoso, sendo responsável por $80 \%$ dos casos; já o adenocarcinoma ocorre de forma mais rara, atinge o epitélio glandular e é responsável por $10 \%$ dos casos. Esta patologia desenvolve-se lentamente, inicialmente pode causar sintomas e evoluir para sangramentos vaginais após a relação sexual, leucorreia anormal, dores abdominais intensas e queixas urinárias (Dantas, Leite, César, Silva, Souza \& Nascimento, 2018).

A periodicidade da realização do exame preventivo, estabelecida pelo Ministério da Saúde está de acordo com as recomendações dos principais programas internacionais de controle do câncer que traz: a faixa etária que deve-se ter como prioridade para realização do exame é entre 25 a 60 anos de idade, sendo realizado uma vez por ano e, após dois exames anuais consecutivos com resultados negativos, a cada três anos, torna--se importante ressaltar que toda mulher que já teve sua primeira coitarca deve submeter-se ao exame até os 64 anos de idade (Barbosa, Silva, Silva, Costa \& Santos, 2017).

Apesar da implantação de programas pelo Ministério da Saúde, como o Programa Nacional de Controle do Câncer do Colo do Útero e a ampla divulgação das informações a respeito do exame preventivo na rede básica de saúde, estudos mostram que ainda existem muitas mulheres que contém um conhecimento inadequado acerca do exame preventivo do CCU (Silva, Freitas, Müller \& Magalhães, 2021).

É imprescindível que os profissionais de saúde, principalmente enfermeiros, voltem seu olhar para essa realidade, pois a morbimortalidade por CCU pode ser reflexo de ações e políticas de prevenção deficitárias. Além disso, vencer as barreiras para uma melhor adesão da mulher ao exame preventivo Papanicolau significa dar atenção aos relatos e experiências de quem faz o exame para identificar o significado deste para as mulheres que a ele se submetem, de modo a extrair informações e argumentos para planejar e adequar às orientações de prevenção deste câncer, uma vez que muitas mulheres sentem-se receosas para realização do exame, além da inclusão de vários fatores que interferem a sua realização (Sousa \& Miranda, 2018).

Diante deste contexto, o presente estudo tem como objetivo descrever a percepção de mulheres acerca da prevenção do câncer de colo do útero e a realização do exame Papanicolau. 


\section{Metodologia}

O presente estudo trata-se de uma revisão integrativa da literatura, sendo definida como um tipo de investigação voltada para o aspecto qualitativo de uma determinada questão, neste caso, a percepção de mulheres acerca da prevenção do câncer de colo do útero e a realização do exame Papanicolau. A revisão de literatura permite aprofundar dentro de diversos autores e referenciais, sobre os discursos e principais temas abordados (Pereira et al., 2018).

Para a revisão integrativa da literatura foram percorridas as seguintes etapas: identificação do tema e seleção da questão norteadora, estabelecimento de critérios de inclusão e exclusão de estudos, categorização e avaliação dos artigos incluídos na revisão, interpretação dos resultados e a síntese do conhecimento dos principais resultados evidenciados na análise dos artigos incluídos (Mendes, Silveira, \& Galvão, 2008).

O tema, determinou a construção da estratégia PICo, que representa um acrômio para Paciente ou Problema (P), Interesse (I) e Contexto (Co). A utilização dessa estratégia para formular a questão norteadora na condução de métodos de revisão possibilita a identificação de palavras-chaves, auxiliando na identificação de estudos primários relevantes nas bases de dados pesquisada (Melnyk \& Fineout-overholt, 2011). Assim, foi utilizada para a geração da questão norteadora desta revisão integrativa da literatura: "Qual a percepção das mulheres acerca da prevenção do câncer de colo do útero e a realização do exame Papanicolau?".

O levantamento do estudo foi realizado entre julho e agosto de 2021, onde a busca dos artigos foi realizada através das bases de dados Medical Literature Analysis and Retrieval System Online (MEDLINE), Literatura Latino-Americana e do Caribe em Ciências da Saúde (LILACS) e Base de Dados de Enfermagem (BDENF).

Para a localização dos estudos relevantes, que respondessem à pergunta da pesquisa, utilizou-se os descritores: "Neoplasias do Colo do Útero", "Teste de Papanicolaou" e "Saúde da Mulher", localizados na lista dos Descritores em Ciências da Saúde (DeCS), disponíveis no portal da Biblioteca Virtual em Saúde em (http://decs.bvs.br/). Tais descritores foram cruzados utilizando os operadores booleanos "AND" para obtenção dos critérios de inclusão e exclusão.

Os critérios de inclusão estabelecidos para a seleção dos artigos foram: artigos completos publicados nas bases de dados elencadas, nos idiomas português e inglês, com recorte temporal de 2015 a 2021 e relacionados com a temática. Foram excluídos os estudos duplicados, incompletos, cartas ao editor, resumos, resenhas, monografias, dissertações, teses, e artigos publicados em anais de eventos, dentre aqueles que estão fora do recorte temporal.

\section{Resultados}

Foram identificados 348 artigos nas bases de dados. Após aplicação dos critérios de inclusão e exclusão, 244 foram excluídos, restando 104 artigos. Após a leitura dos títulos e resumos, foram excluídos 72 artigos, resultando num total de 32 publicações selecionadas. Destas, 06 foram excluídos por estarem duplicados nas bases de dados elencadas, resultando em 26 estudos. Após a leitura na integra, 10 estudos foram excluídos por não responderem à questão norteadora, resultando em 16 artigos que foram selecionados para compor a presente revisão. O fluxograma com o detalhamento das etapas de pesquisa está apresentado a seguir na Figura 1. 
Figura 1. Fluxograma de identificação e seleção dos artigos, 2021.

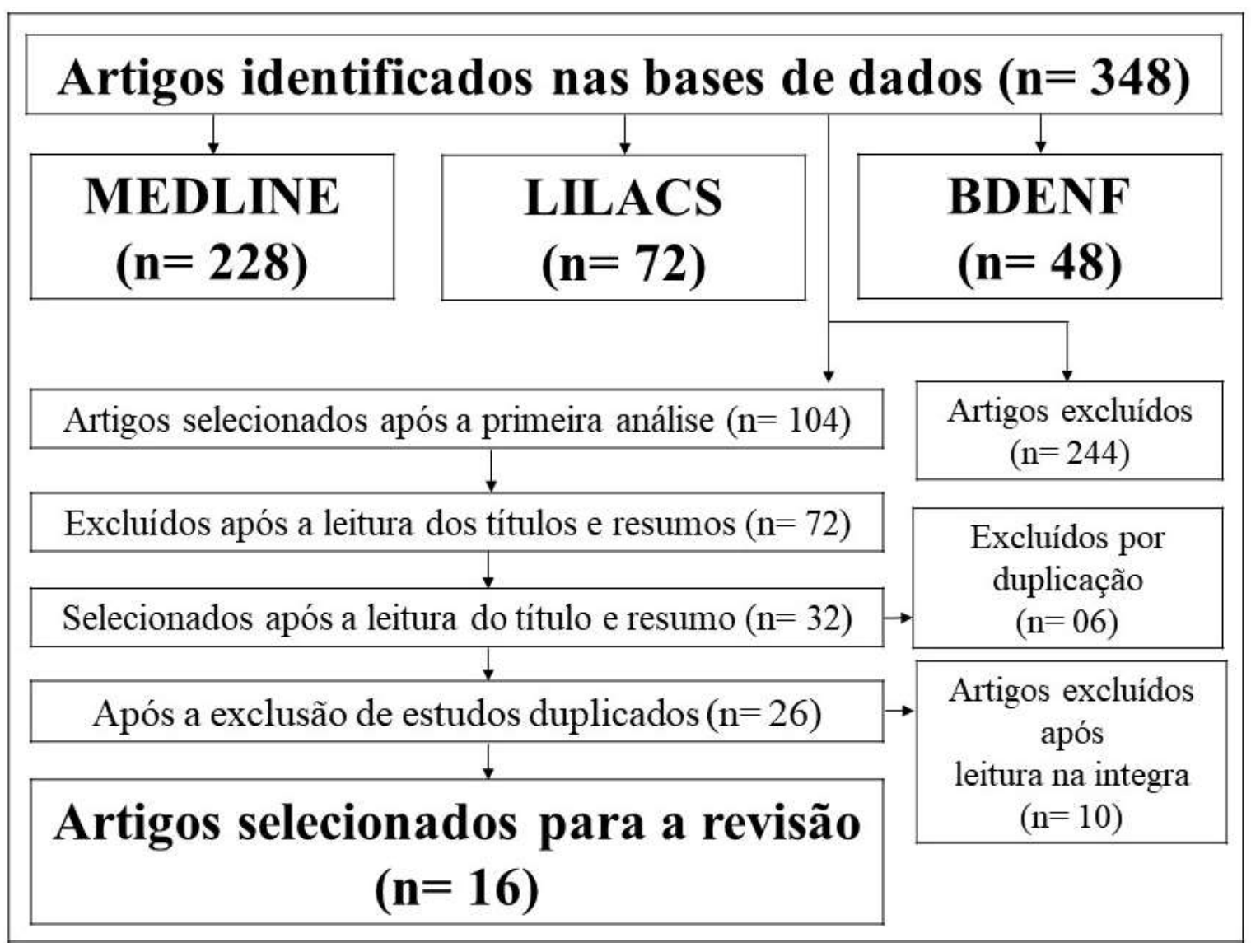

Fonte: Autores (2021).

Na Tabela 1 apresenta os estudos selecionados para a composição da presente revisão integrativa da literatura de forma mais detalhada, seguido as informações como título do artigo, autores e ano de publicação, objetivo e principais resultados. 
Tabela 1. Distribuição dos artigos selecionados de acordo com o título, autores e ano de publicação, objetivos e principais resultados, 2021.

\begin{tabular}{ll}
\hline $\mathbf{N}^{\mathbf{0}}$ & \multicolumn{2}{c}{ TÍTULO } \\
\hline 01 & $\begin{array}{l}\text { The Elderly Women's Perception of } \\
\text { Cervical Cancer Preventio } \\
\text { Examination }\end{array}$ \\
02 & $\begin{array}{l}\text { Adesão ao exame Papanicolau po } \\
\text { mulheres jovens em Unidad }\end{array}$
\end{tabular}
mulheres jovens em Unidade Básica de Saúde

03 Exame Papanicolau: fatores que influenciam a não realização do exame em mulheres de 40 a 65 anos

04 Conhecimento e prática de mulheres atendidas na atenção primária a saúde sobre o exame Papanicolaou

05 Exame preventivo do câncer de colo do útero: conhecimento de

Mulheres

06 Câncer de colo uterino: conhecimento, atitude e prática sobre o exame de prevenção

\section{ATORES/ANO \\ Leite, Nunes, Oliveira, \\ Barbosa, Souza \& Teles}

(2019)

Silva, Lessa, Silva, Albuquerque, Ferreira \& Silva (2016)

Silva et al.,

\section{OBJETIVO}

Descrever a percepção das

mulheres idosas sobre o exame

preventivo do câncer de colo de útero (PCCU)

Investigar os motivos do não comparecimento ao exame preventivo Papanicolau das mulheres

jovens que realizaram o exame

\section{Caracterizar os fatores que} influenciam

mulheres de 40 a 65 anos de idade a não realizarem o exame

$$
\text { Papanicolau }
$$

Silva, Freitas, Müller \& Magalhães (2021)

Albuquerque, Miranda, Leite \& Leite

(2016)

Melo, Linhares, Silva, Pontes, Silva \& Oliveira (2019)
Avaliar o conhecimento e a prática de mulheres atendidas em Unidades Básica de Saúde em relação ao exame Papanicolaou

Analisar o conhecimento de mulheres acerca do exame preventivo do câncer de colo do útero (CCU), bem como verificar a prática do exame.

Avaliar o conhecimento, atitude e prática de mulheres sobre o rastreamento do câncer cérvicouterino e investigar sua associação com variáveis sociodemográficas

\section{PRINCIPAIS RESULTADOS}

A maioria das idosas entrevistadas possui o entendimento empírico sobre o exame preventivo, considerando-o importante, todavia muitas delas indagam sentimentos de vergonha e medo ao se submeterem ao exame. A orientação quanto à periodicidade da realização do preventivo é feita pelos profissionais de saúde, porém muitas não o realizam.

O estudo mostrou que os motivos apresentados pelas mulheres jovens, para a não repetição do exame citopatológico, foi acreditarem não precisar realizar o Papanicolau por estarem bem de saúde.

Constatou-se no estudo que as mulheres tendem a realizar o Papanicolau anualmente, mas existem fatores que influenciam a não realização do exame. Os motivos mais relatados são vergonha, medo e medo do diagnóstico.

O estudo evidencia a existência conhecimento precário da maioria das mulheres sobre o exame preventivo do Câncer do Colo do Útero, tendo como consequência uma atribuição errônea sobre a finalidades do mesmo.

As mulheres pesquisadas possuíam algum conhecimento acerca do exame preventivo do CCU sendo, este conhecimento, superficial.

Apresentaram esclarecimento satisfatório sobre os cuidados antes da realização

desse exame $(93,3 \%)$. A maioria $(86,67 \%)$ declarou já ter recebido informações sobre o exame e todas consideraram necessária a realização do mesmo.

A pesquisa aponta que as mulheres realizam o exame, julgam necessário, mas não possuem conhecimentos adequados, o que demonstra a necessidade de ações educativas por parte do enfermeiro e demais profissionais de saúde. 
07 Acesso ao exame citológico do colo do útero em região de saúde: mulheres invisíveis e corpos vulneráveis

08 Barreiras à realização do exame Papanicolau: perspectivas de usuárias e profissionais da Estratégia de Saúde da Família da cidade de Vitória da Conquista BA.

09 Percepção de usuárias acerca do exame de detecção precoce do câncer de colo uterino

10 Percepção e adesão das mulheres quanto ao exame citopatológico

11 Percepção de mulheres sobre o teste de Papanicolaou

12 Fatores relacionados a não adesão à realização do exame de Papanicolau

13 Implication of violence against women on not performing the cytopathologic test

14 Educação popular em saúde como estratégia à adesão na realização do exame colpocitológico
Fernandes, Galvão, Assis, Almeida \& Santos

Aguiar \& Soares (2015)

Neves, Oliveira, Galvão,

Ferreira, Mangane \& Sousa

Miranda, Rezende \&

Romero

(2018)

Silva, Oliveira \& Vargens (2016)

Silva, Teixeira, Ferrari, Cestari \& Cardelli

Leite, Amorim \& Gigante (2018)

Alves, Alves, Assis (2016)
Avaliar o acesso ao exame citológico do câncer do útero na Estratégia Saúde da Família

(ESF), em municípios de uma região de saúde da Bahia.

Conhecer as barreiras que levam mulheres em idade fértil da cidade de Vitória da Conquista -

BA a não realizarem o exame

Papanicolau, na perspectiva das próprias mulheres e dos profissionais de saúde. descrever a percepção de usuárias acerca do exame de detecção precoce do câncer de colo uterino

Conhecer a concepção das mulheres sobre o exame

Papanicolau, e os fatores relacionados a não adesão ao exame.

Desvelar a percepção de mulheres sobre o Teste de Papanicolaou

identificar motivos para baixa adesão ao exame de

Papanicolau entre mulheres atendidas na atenção primária de saúde

Analisar a associação entre a violência por parceiro íntimo e não realização do exame

citopatológico nos últimos três anos

Apresentar a experiência com educação popular em saúde como metodologia ativa de
Os resultados indicam que residir em zona rural é uma barreira para o acesso ao exame Papanicolaou e reforça as iniquidades, e que os enfermeiros são a principal referência para a realização do exame preventivo.

O estudo aponta as seguintes barreiras para não realização do exame: conhecimento insuficiente, sentimentos negativos, falta de atitude, aspectos vinculados aos serviços de saúde e inserção da mulher no mercado de trabalho.

O estudo relata que a realização do exame de CCU por profissional de saúde do sexo masculino pode constituir motivo de recusa do exame.

Foi evidenciado que as mulheres tem conhecimento acerca da importância do exame preventivo de CCU, apesar dos sentimentos negativos que apresentam devido ao exame.

As mulheres percebem o exame Papanicolaou como desconfortável, mas entendem a necessidade e a importância do cuidado à saúde.

O estudo aponta que embora o rastreamento do câncer de colo de útero seja fundamental para intervenção a tempo oportuno, significativa parcela das mulheres ainda não adere ao exame por mitos e tabus, crenças e atitudes em saúde, bem como organização do serviço.

O estudo conclui que a violência apresenta-se como um agravo importante e com impacto negativo na saúde da mulher. Mulheres vitimizadas, física ou sexualmente, por seus companheiros, estão mais vulneráveis a não realização do exame de Papanicolaou e, consequentemente, têm menos oportunidades de detecção precoce do câncer de colo do útero.

Constatou-se que estratégias de educação popular em saúde junto à comunidade podem permitir melhor adesão à realização do exame citopatológico do colo do útero, uma das estratégias públicas mais 
15 Conhecimento de idosas sobre o exame citopatológico fatores da não adesão acerca do exame Papanicolau
Santos, Cordeiro, Braga Moraes, Araújo \& Dias

Dantas, Leite, César, Silva,

Souza \& Nascimento (2018) aprendizagem, desenvolvida por uma equipe de Estratégia de Saúde de Família (ESF) da grande Porto Alegre, na adesão das mulheres à realização do

exame colpocitológico

efetivas, seguras e de baixo custo para detecção precoce desse tipo de câncer, o que pode contribuir para a redução da incidência de novos casos nessa comunidade.

Investigar o conhecimento das mulheres de um grupo de idosas sobre o exame ginecológico e averiguar o nível de entendimento das mulheres idosas sobre o HPV e suas consequências

Averiguar o conhecimento das mulheres sobre o Papanicolau

\section{A pesquisa evidencia que todas as mulheres do estudo conhecem o exame Papanicolau, mas nem todas sabem de sua principal função. $\mathrm{O}$ principal fator para não o realizarem é vergonha e falta de orientação, a maior parte o realiza anualmente e a maioria não recebe orientações da} enfermeira.
A maioria das idosas entrevistadas possui o entendimento empírico sobre o exame ginecológico, considerando-o importante, porém não o realizam periodicamente. Em relação ao HPV, todas as idosas o desconhecem completamente.

Fonte: Pesquisa realizada (2021) 


\section{Discussão}

No Brasil, o número de casos diagnosticados em estágio avançado de CCU ainda é muito alto, corroborando os dados de outros países em desenvolvimento, que mostram a baixa eficácia das campanhas de detecção precoce. Considerando ainda que este câncer é uma das doenças que mais afetam as mulheres e aumentam com a longevidade feminina, dar-se a importância desse exame ser enfatizado, principalmente, pelos programas específicos para a saúde da mulher (Leite, Nunes, Oliveira, Barbosa, Souza \& Teles, 2019).

O CCU costuma manifestar-se sob algumas condições como: a partir da faixa etária de 20 a 29 anos, tendo um aumento de seu risco rapidamente até atingir o pico etário entre 50 e 60 anos. Tendo como fator de risco para o aparecimento do CCU, e o principal, a infecção pelos Vírus do Papiloma Humano genital oncogênico (HPV), com alguns subtipos de alto risco e relacionados a tumores malignos (Silva, Lessa, Silva, Albuquerque, Ferreira \& Silva, 2016).

Atualmente, as mulheres buscam realizar o exame citopatológico como uma forma de manutenção da sua saúde, objetivando seu bem-estar físico, uma vez que lesões graves se tornam mais frequentes nas mulheres com mais idades, principalmente a partir dos 45 anos. Desta forma, a realização periódica nessa faixa etária torna-se essencial para a realização do exame Papanicolau (Silva et al., 2018).

Silva, Freitas, Müller \& Magalhães (2021) relatam que há diversos fatores associados a não realização do exame, destacando-se os baixos níveis de escolaridade; a baixa renda familiar; o uso de contraceptivo oral e aspectos relacionados ao serviço de saúde como dificuldade para marcar consulta, disponibilidade do serviço e demora no resultado. Outros fatores que se destacam e geralmente um dos mais influenciadores é o déficit de conhecimento referente a importância e finalidade do exame e a falta de interesse para realização do mesmo.

Para a realização do exame de Papanicolau alguns cuidados necessários antes de sua realização devem ser necessários. Dentre eles estão: abster-se de relação sexual por três dias antes do exame, realizar higiene, não usar cremes e estar no dia do ciclo menstrual correto (Albuquerque, Miranda, Leite \& Leite, 2016).

Melo, Linhares, Silva, Pontes, Silva \& Oliveira (2019) citam que as mulheres têm atitude adequada quanto à necessidade de realização do exame Papanicolau, apresentando uma prática adequada, pois fazem o exame, buscam o resultado e mostram para o profissional de saúde, apesar de apresentarem um conhecimento insuficiente, uma vez que algumas mulheres nunca ouviram falar do exame de prevenção, ou já ouviram falar, mas não sabem sua finalidade e/ou não são capazes de citar um cuidado indispensável para sua realização e/ou sua periodicidade. Vista a isso, muitas mulheres realizam o exame devido solicitação dos profissionais das equipes de saúde, e estes, por sua vez, não promovem o conhecimento adequado por meio da educação em saúde, principalmente os profissionais enfermeiros e os agentes comunitários de saúde (ACS) (Fernandes, Galvão, Assis, Almeida \& Santos, 2019).

Leite, Nunes, Oliveira, Barbosa, Souza \& Teles (2019) também citam em seu estudo que a falta de conhecimento acerca da importância e da finalidade do exame preventivo do CCU está associada a baixa escolaridade, visto que a escolaridade está diretamente relacionada ao conhecimento e adesão ao exame. Ademais, o baixo nível socioeconômico de algumas mulheres também contribui para tal situação, pois à medida que diminui o nível socioeconômico, aumenta significativamente a prevalência de mulheres sem cobertura pelo exame Papanicolau (Aguiar \& Soares, 2015).

Além disso, a forma como o exame de Papanicolau é realizado, devido exposição da genitália, tabus e o conhecimento inadequado sobre seu câncer ginecológico, a sua realização produz desconforto emocional e outros sentimentos na mulher, bem como: medo do resultado, ansiedade, nervosismo, vergonha, frustração e desconforto em realiza-lo, estes acabam tornando-se fatores que interferem na realização do exame pela mulher (Dantas, Leite, César, Silva, Souza \& Nascimento, 2018). 
Neves, Oliveira, Galvão, Ferreira, Mangane \& Sousa (2016) relatam que a exposição do corpo, principalmente a um profissional de saúde do sexo oposto, constitui fator de constrangimento às mulheres. Contudo, a consciência sobre a importância do exame e o vínculo de confiança estabelecido entre profissional de saúde e usuária minimizam sentimentos negativos provenientes dessa exposição. O modo como algumas mulheres reagem ao expor seu corpo, vê-lo sendo manipulado e examinado por um profissional da saúde, revela o quanto a sexualidade influencia a vida da mulher; afinal, trata-se de tocar, manusear e expor órgãos e zonas erógenas. Assim, surge a questão de as mulheres associarem sempre a exposição das genitálias à sexualidade, o que produz sentimento de vergonha em relação aos seus órgãos sexuais.

É relatado ainda que as mulheres atribuem o exame de Papanicolau como fundamental para a prevenção e detecção precoce para doenças, mesmo havendo referências desagradáveis como: dor, desconforto e até mesmo constrangimento durante a realização do mesmo (Miranda, Rezende \& Romero, 2018), como já citado anteriormente. No entanto, as mulheres o associam à dor e aos desconfortos causados durante o procedimento e, embora o exame preventivo seja normalmente indolor, é importante que a mulher não esteja nervosa e não tenha lesões na parede vaginal. Além disso, o profissional de saúde deve realizá-lo de acordo com a técnica correta (Silva, Oliveira \& Vargens, 2016).

Outra barreira para o não comparecimento ao exame citopatológico é o ciclo menstrual que pode coincidir com o agendamento da coleta inviabilizando a realização do procedimento, portanto, o serviço de saúde deve contar com essa variável para estruturar o programa para aumentar a adesão e cobertura. Nesse sentido, surge a necessidade de maiores investimentos informativos para que as mulheres evitem buscar cuidado na eminência de queixas ginecológica como alteração no ciclo menstrual e sangramento após relação sexual, fazendo uso tardio do exame de citologia oncótica que implica no baixo impacto preventivo, sendo necessário encaminhamento para serviços especializados, devido ao avanço da doença (Silva, Teixeira, Ferrari, Cestari \& Cardelli, 2015).

Evidencia-se que o conhecimento da finalidade do exame de Papanicolau influencia as mulheres a se submeterem ao mesmo, resultando em uma maior e mais consciente procura, enquanto que a desinformação sobre a doença e o exame prejudica a mulher na procura dos cuidados preventivos. A desinformação pode gerar despreocupação e consequente desinteresse pela prevenção, não só do CCU, como também de outras doenças ginecológicas. No entanto, estudos mostram, ainda, que as mulheres se submetem ao exame preventivo quando têm facilidade de acesso ao programa de prevenção de doenças ginecológicas, e também quando possuem algum suporte de convênio médico (Leite, Amorim \& Gigante, 2018). Desta forma, a falta de informação, o conhecimento errôneo ou insuficiente, constituem barreiras para a realização de medidas preventivas para o CCU (Silva, Freitas, Müller \& Magalhães, 2021).

Um dos fatos a ser apontado é a importância de orientar a respeito do uso da vacina contra HPV já no início da adolescência, como é preconizado pelo Ministério da Saúde. Alguns estudos apontam que a vacina contra o HPV é eficaz em sua proteção contra os subtipos oncogênicos dessa doença sexualmente transmissível e, consequentemente, é um fator de prevenção para a neoplasia uterina desde que as doses sejam administradas no tempo correto (Silva, Lessa, Silva, Albuquerque, Ferreira \& Silva, 2016).

Diante disso, há a necessidade de treinamento de profissionais de saúde para o cumprimento das recomendações do Ministério da Saúde quanto ao rastreamento, o que inclui informar a regularidade de exames e facilitar o acesso ao exame preventivo. Além dessas questões técnicas, o profissional deve ter mais sensibilidade e compreensão desse cenário bastante comum na realização do exame, levando em consideração o fato de que a mulher pode deixar de realizá-lo, a depender dos sentimentos envolvidos (Silva, Oliveira \& Vargens, 2016).

Cabe ao enfermeiro atuante nos programas de prevenção e controle do CCU fomentar ações que enfatizem mais a necessidade da realização do exame preventivo para que haja efetivo impacto sobre a morbimortalidade por este câncer. 
Acredita-se que a implantação de um programa bem desenvolvido para essa população seja a melhor estratégia na tentativa de diminuir a incidência das lesões iniciais e mortalidade por CCU, o que facilita a melhoria na qualidade de vida das mulheres (Albuquerque, Miranda, Leite \& Leite, 2016).

Embora o CCU seja uma das principais causas de mortalidade das mulheres no Brasil ainda é baixa a adesão à realização do exame CP. A Estratégia Saúde da Família tem um importante papel para a mudança dessa realidade devendo dar ênfase na qualificação e fortalecimento das ações de promoção da saúde, no âmbito da atenção básica, de forma a estimular o protagonismo das mulheres para o autocuidado e a prevenção do câncer do colo uterino (Alves, Alves, Assis, 2016).

Diante do exposto, a educação em saúde torna-se uma excelente aliada no combate às neoplasias do colo do útero, pois combina oportunidades que contribuem com a manutenção da saúde e sua promoção, ultrapassando a tradicional transmissão de conteúdos e aproximando os casos à realidade do usuário por meio da exemplificação de práticas educativas e o incentivo a autonomia, atribuindo ao indivíduo responsabilidade sobre sua condição de saúde, e o empoderamento necessário para o pleno exercício de construção da cidadania (Santos, Cordeiro, Braga, Moraes, Araújo \& Dias, 2015).

\section{Considerações Finais}

O estudo possibilitou identificar que o conhecimento das mulheres acerca do exame Papanicolau ainda se encontra incipiente, havendo vários fatores que fazem com que elas o deixem de realizar, ocasionando numa menor chance de cura, caso o diagnóstico seja positivo. Assim, é evidente que a percepção das mulheres diante do exame de detecção precoce do CCU é de fundamental importância para a adesão ao mesmo. No entanto, as mulheres podem apresentar sentimentos negativos que podem interferir na realização do exame, como: medo, desconforto, vergonha, insegurança, nervosismo, ansiedade, dentre outros.

Neste sentido, é indispensável as orientações pelos profissionais da saúde sobre o exame Papanicolau às mulheres que buscam os serviços de atenção básica. Devendo estes, realizar ações de educação em saúde de forma continuada, enfatizando a importância da realização periódica do exame Papanicolau pelas mulheres, proporcionando uma maior adesão ao exame, além de possibilitar uma assistência humanizada, contínua e de qualidade, até mesmo estratégias que busque a curiosidade da mulher em conhecer o seu próprio corpo e sua saúde.

Por fim, sugere-se que futuros trabalhos abordem as ações que os profissionais da saúde possam realizar, com o intuito de incentivar a mulher a procurar os sistemas de saúde e realizar os exames preventivos de CCU periodicamente, assim, proporcionando um melhor rastreamento por parte dos sistemas na detecção precoce deste câncer que ainda encontra-se prevalente na população feminina.

\section{Referências}

Aguiar, R. P. \& Soares, D. A. (2015). Barreiras à realização do exame Papanicolau: perspectivas de usuárias e profissionais da Estratégia de Saúde da Família da cidade de Vitória da Conquista - BA. Physis: Revista de Saúde Coletiva, 25(2): 359-379.

Albuquerque, V. R., Miranda, R. V., Leite, C. A. \& Leite, M. C. A. (2016). Exame preventivo do câncer de colo do útero: conhecimento de mulheres. Rev enferm UFPE on line., 10(Supl. 5) :4208-18.

Alves, S. R., Alves, A. O. \& Assis, M. C. S. (2016). Educação popular em saúde como estratégia à adesão na realização do exame colpocitológico. Ciência, Cuidado \& Saúde, 15(3), 570-574.

Barbosa, L. C. R., Silva, C. M. A., Silva, D. A., Costa, L. J. S. F. \& Santos, N. R. (2017). Percepção de mulheres sobre os fatores associados a não realização do exame Papanicolau. Interfaces Científicas - Saúde e Ambiente, 5(3): 87-96.

Colares, W. T. H. C. et al. (2020). Análise clínico-epidemiológica do Câncer de colo uterino em Manaus: relação entre faixa etária e estadiamento/ clinicalepidemiological analysis of cervical cancer in manaus. Brazilian Journal Of Health Review, 3(6): 16510-16517.

Dantas, P. V. J., Leite, K. N. S., César, E. S. R., Silva, S. C. R., Souza, T. A. \& Nascimento, B. B. (2018). Conhecimento das mulheres e fatores da não adesão 
acerca do exame Papanicolau. Rev enferm UFPE on line., 12(3): 684-91.

Fernandes, N. F. S. Galvão, J. R., Assis, M. M. A., Almeida, P. F. \& Santos, A. M (2019). Acesso ao exame citológico do colo do útero em região de saúde: mulheres invisíveis e corpos vulneráveis. Cadernos de Saúde Pública, 35(10), e00234618: 01-19.

Gurgel, L. C. et al. (2019). Percepção de mulheres sobre o exame de prevenção de colo de útero Papanicolau: Uma Revisão Integrativa da Literatura. Id on Line Rev. Mult. Psic. 13(46): 434-445.

Leite, B. O., Nunes, C. R. O., Oliveira, V. V., Barbosa, R. A. A., Souza, M. S. \& Teles, M. A. B. (2019). The Elderly Women's Perception of Cervical Cancer Prevention Examination. Rev Fund Care Online, 11(5):1347-1352.

Leite, F. M. C., Amorim, M. H. C. \& Gigante, D. P. (2018). Implication of violence against women on not performing the cytopathologic test. Revista de Saúde Pública, 52(89): 01-10.

Melo, E. M. F., Linhares, F. M. P., Silva, T. M., Pontes, C. M., Santos, A. H. S. \& Oliveira, S. C. (2019). Cervical cancer: knowledge, attitude and practice on the prevention examination. Revista Brasileira de Enfermagem, 72(Supl. 3), 25-31.

Melnyk, B. M., \& Fineout-Overholt, E. (Eds.). (2011). Evidence-based practice in nursing \& healthcare: A guide to best practice. Lippincott Williams \& Wilkins.

Mendes, K. D. S., Silveira, R. C. C. P. \& Galvão, C. M. (2008). Revisão integrativa: método de pesquisa para a incorporação de evidências na saúde e na enfermagem. Texto \& Contexto - Enfermagem. 17(4), p. 758-764.

Miranda, A. P., Rezende, E. V. \& Romero, N. S. A. (2018). Percepção e adesão das mulheres quanto ao exame citopatológico. Revista Nursing, 21(246): 2435-2438.

Neves, K. T., Oliveira, A. W. N., Galvão, T. R. A. F., Ferreira, I. T., Mangane, E. M. \& Sousa, L. B. (2016). Percepção de usuárias acerca do exame de detecção precoce do câncer de colo uterino. Cogitare Enferm., 21(4): 01-07.

Pereira, A. S. (2018). Metodologia da pesquisa científica. UFSM. https://repositorio.ufsm.br/bitstream/handle/1/15824/Lic_Computacao_MetodologiaPesquisa-Cientifica.pdf?sequence $=1$.

Santos, R. F. A., Cordeiro, C. A., Braga, L. S., Moraes, M. N., Araújo, V. S. \& Dias, M. D. (2015). Conhecimento de idosas sobre o exame citopatológico. Rev enferm UFPE on line., 9(2): 517-25.

Santos, T. L. S., Silveira, M. B. \& Rezende, H. H. A. (2019). A importância do exame citopatológico na prevenção do câncer do colo uterino. Enciclopédia biosfera, Centro Científico Conhecer, 16(29): 1-15.

Silva, C. M., Oliveira, D. S., \& Vargens, O. M. C. (2016). Percepção de mulheres sobre o teste Papanicolau. Revista Baiana de Enfermagem, Salvador, 30(2): p. 1-9.

Silva, J. P. et al. (2018). Exame Papanicolau: fatores que influenciam a não realização do exame em mulheres de 40 a 65 anos. Arq. Ciênc. Saúde, 25(2): 15 19.

Silva, L. A., Freitas, A. S., Müller, B. C. T. \& Magalhães, M. J. S. (2021). Conhecimento e prática de mulheres atendidas na atenção primária a saúde sobre o exame Papanicolaou. R. pesq.: cuid. fundam. Online, 13:1013-1019.

Silva, L. S. R., Lessa, E. C., Silva, T. M., Albuquerque, A. K. D. S., Ferreira, M. D. R. \& Silva, T. L. L. (2016). Adesão ao exame Papanicolau por mulheres jovens em unidade básica de saúde. Rev enferm UFPE on line., 10(12): 4637-45.

Silva, M. A. S., Teixeira, E. M. B., Ferrari, R. A. P., Cestari, M. E. W. \& Cardelli, A. A. M. (2015). Fatores relacionados a não realização do exame de Papanicolau. Rev Rene, 16(4):532-539.

Sousa, K. R. \& Miranda, M. A. L. (2018). Câncer do colo do útero: percepção das mulheres frente ao exame preventivo. Com. Ciências Saúde. 29(3):183-190.

Souza, A. F. \& Costa, L. H. R. (2015). Conhecimento de Mulheres sobre HPV e Câncer do Colo do Útero após Consulta de Enfermagem. Revista Brasileira de Cancerologia, 61(4): 343-350. 